EPJ Web of Conferences 43, 05013 (2013)

DOI: $10.1051 /$ epjconf/20134305013

(C) Owned by the authors, published by EDP Sciences, 2013

\title{
GD 154: White dwarf with multi- and monoperiodic pulsation
}

\author{
M. Paparóa and Zs. Bognár \\ Konkoly Observatory, MTA CSFK, Konkoly-Thege M. u. 15-17, 1121 Budapest, Hungary
}

\begin{abstract}
We present the white dwarf GD 154 as an example where either monoperiodic or multiperiodic pulsation were found at different epochs. The mono-multi-monoperiodic stage seems to alternate. Many questions have been raised. Is this behaviour connected to the evolution of DAV stars? How often does it happen? Is there any regularity in this change of the pulsational behaviour or is it irregular?
\end{abstract}

\section{INTRODUCTION}

The light variation of g-mode white dwarf pulsators shows diversity from the simple sinusoidal to the mightly complicated one. Examples can be found for both monoperiodic and complex multiperiodic behaviour. The variability of the frequency content, especially due to amplitude variability of the excited modes is well-established. We believe that most of the white dwarfs exist in one or the other pulsational stage for a long time.

\section{MONOPERIODIC PULSATOR AT DISCOVERY}

GD 154 is one of the three DAV white dwarfs that were discovered as a monoperiodic pulsator with harmonics $(2 \mathrm{~F}, 3 \mathrm{~F}, 4 \mathrm{~F}, 5 \mathrm{~F})$ and intermediate frequencies $(1.52 \mathrm{~F}, 2.53 \mathrm{~F}, 3.53 \mathrm{~F})$ ([1]; left panel in Fig. 1). The intermediate frequencies, the "second series" are close to, but distinctly different from the odd halfinteger harmonics of the main frequency. The simplicity of these stars broke the amplitude-complexity correlation in DAV stars and suggested a filter mechanism in the DBV objects. The dominant mode $(72.839 \mathrm{c} / \mathrm{d})$ has large amplitude in comparison with the harmonics and the near half-integer frequencies' values.

\section{MORE AND MORE MODES ARE EXCITED}

New observing runs revealed a new structure of the pulsation in GD 154. The right panels of Fig. 1 show the independent excited modes of the WET campaign ([2]; upper panel) and the Konkoly observing run over a six-month timebase in 2006 (bottom panel). The values of an additional mode out of the plotted range are given in the panels. This mode is close to the second harmonic of the dominant mode. Possible rotational triplets and doublets are also marked. In 1991 three independent modes and many linear combinations, in 2006 six independent modes but no combination frequencies were found compared to the original monoperiodic pulsational stage. The dominant frequency was close in 1991 (72.819 c/d) to the discovery value. However, the dominant mode's value changed in 2006 to the lower frequency member of the rotational triplet $(72.502 \mathrm{c} / \mathrm{d})$. The amplitude of the dominant frequency was remarkably lower in 2006 (8.4 mmag) than in 1977 (34 mmag).

\footnotetext{
a e-mail: benko@konkoly.hu
}

This is an Open Access article distributed under the terms of the Creative Commons Attribution License 2.0, which permits unrestricted use, distribution, and reproduction in any medium, provided the original work is properly cited. 
EPJ Web of Conferences
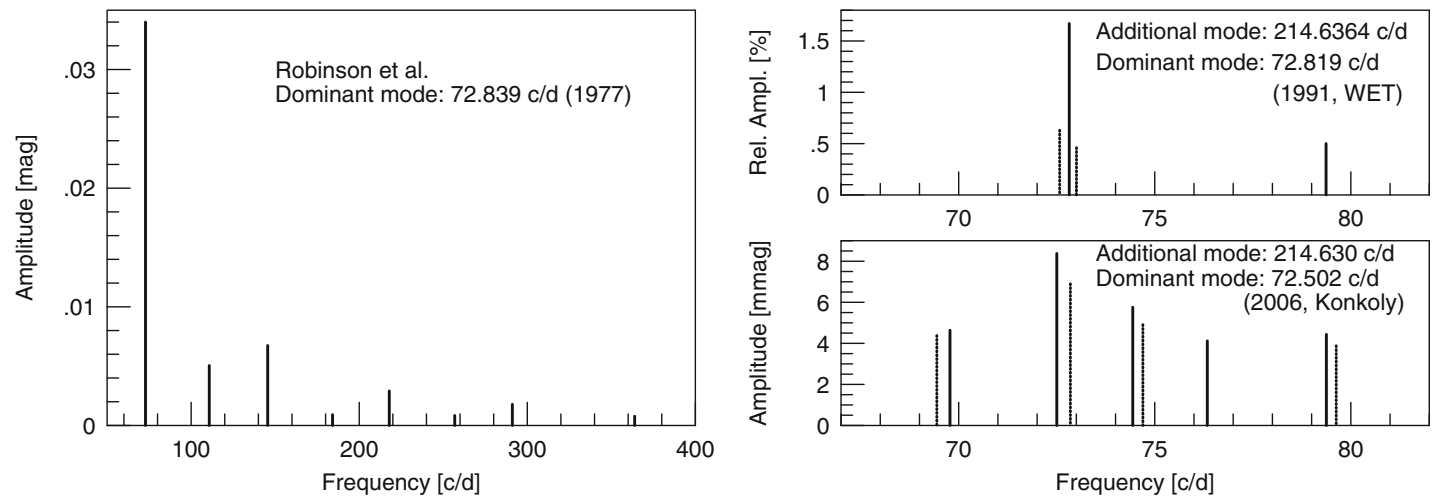

Figure 1. Left: The scematic Fourier spectrum at discovery. Right: Three modes were excited in 1991, six modes in 2006 .

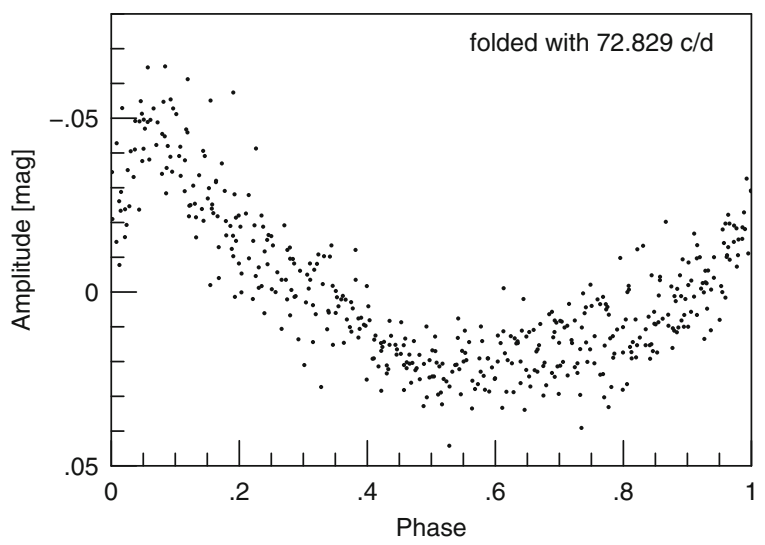

Figure 2. Folded light curve at the end of the run in 2006.

\section{NEW PULSATIONAL PHASE STARTED}

The "forte" [3] or "sforzando" [4] effect in GD 358 was a case when a multimode pulsation changed to a monoperiodic one on a timescale of hours with remarkable amplitude increase.

The last subset of the observing run in 2006 showed the change of the pulsation back to monoperiodic. Figure 2 shows the light curves folded with the new dominant frequency. The regularity with the new value which is similar to the discovery one, is obvious. A new, complete observation of a monoperiodic stage might help to answer the questions whether the mono-multi-monoperiodic pulsation appears regularly and what the timescale of the changes is.

\section{References}

[1] Robinson, E.L., Stover, R., Nather, R.E., McGraw, J.T., ApJ 220, (1978) 614-619

[2] Pfeiffer, B., Vauclair, G., Dolez, N. et al., A\&A 314, (1996) 182-190

[3] Kepler, S.O., Nather, R.E., Winget, D.E. et al., A\&A 401, (2003) 639-654

[4] Provencal, J.L., Montgomery, M.H., Kanaan, A. et al., ApJ 693, (2009) 564-585 